Comment. Math. Helv. 79 (2004) 350-361 0010-2571/04/020350-12

DOI 10.1007/s00014-003-0797-2 (c) 2004 Birkhäuser Verlag, Basel

Commentarii Mathematici Helvetici

\title{
On the zero set of semi-invariants for tame quivers
}

Christine Riedtmann and Grzegorz Zwara

\begin{abstract}
Let $\mathbf{d}$ be a prehomogeneous dimension vector for a finite tame quiver $Q$. We show that the common zeros of all non-constant semi-invariants for the variety of representations of $Q$ with dimension vector $N \cdot \mathbf{d}$, under the product of the general linear groups at all vertices, is a complete intersection for $N \geq 3$.
\end{abstract}

Mathematics Subject Classification (2000). 14L24, 16G20.

Keywords. Semi-invariants, quivers, representations.

\section{Introduction}

Let $k$ be an algebraically closed field, and let $Q=\left(Q_{0}, Q_{1}, t, h\right)$ be a finite quiver, i.e. a finite set $Q_{0}=\{1, \ldots, n\}$ of vertices and a finite set $Q_{1}$ of arrows $\alpha: t \alpha \rightarrow h \alpha$, where $t \alpha$ and $h \alpha$ denote the tail and the head of $\alpha$, respectively.

A representation of $Q$ over $k$ is a collection $\left(X(i) ; i \in Q_{0}\right)$ of finite dimensional $k$-vector spaces together with a collection $\left(X(\alpha): X(t \alpha) \rightarrow X(h \alpha) ; \alpha \in Q_{1}\right)$ of $k$-linear maps. A morphism $f: X \rightarrow Y$ between two representations is a collection $(f(i): X(i) \rightarrow Y(i))$ of $k$-linear maps such that

$$
f(h \alpha) \circ X(\alpha)=Y(\alpha) \circ f(t \alpha) \quad \text { for all } \alpha \in Q_{1} .
$$

By $\sigma(X)$ we denote the number of pairwise non-isomorphic indecomposable direct summands occurring in a decomposition of $X$ into indecomposables. According to the theorem of Krull-Schmidt, $\sigma(X)$ is well-defined. The dimension vector of a representation $X$ of $Q$ is the vector

$$
\operatorname{dim} X=(\operatorname{dim} X(1), \ldots, \operatorname{dim} X(n)) \in \mathbb{N}^{Q_{0}} .
$$

We denote the category of representations of $Q$ by $\operatorname{rep}(Q)$, and for any vector $\mathbf{d}=\left(d_{1}, \ldots, d_{n}\right) \in \mathbb{N}^{Q_{0}}$

$$
\operatorname{rep}(Q, \mathbf{d})=\prod_{\alpha \in Q_{1}} \operatorname{Mat}\left(d_{h \alpha} \times d_{t \alpha}, k\right)
$$


is the vector space of representations $X$ of $Q$ with $X(i)=k^{d_{i}}, i \in Q_{0}$. The group

$$
\mathrm{Gl}(\mathbf{d})=\prod_{i=1}^{n} \mathrm{Gl}\left(d_{i}, k\right)
$$

acts on $\operatorname{rep}(Q, \mathbf{d})$ by

$$
\left(\left(g_{1}, \ldots, g_{n}\right) \star X\right)(\alpha)=g_{h \alpha} \cdot X(\alpha) \cdot g_{t \alpha}^{-1} .
$$

Note that the $\mathrm{Gl}(\mathbf{d})$-orbit of $X$ consists of the representations $Y$ in $\operatorname{rep}(Q, \mathbf{d})$ which are isomorphic to $X$.

We call $\mathbf{d}$ a prehomogeneous dimension vector if $\mathrm{Gl}(\mathbf{d}) \star T$ is an open orbit for some $T$ in $\operatorname{rep}(Q, \mathbf{d})$. Such a representation $T$ is characterized by $\operatorname{Ext}_{Q}^{1}(T, T)=0$ [9]. If $Q$ admits only finitely many indecomposable representations, or equivalently if the underlying graph of $Q$ is a disjoint union of Dynkin diagrams of type $\mathbb{A}, \mathbb{D}$ or $\mathbb{E}[6]$, every vector $\mathbf{d}$ is prehomogeneous. Indeed, any representation is a direct sum of indecomposables and therefore $\operatorname{rep}(Q, \mathbf{d})$ contains finitely many orbits, one of which must be open.

Let $\mathbf{d}$ be prehomogeneous, and let $f_{1}, \ldots, f_{s} \in k[\operatorname{rep}(Q, \mathbf{d})]$ be the irreducible monic polynomials whose zeros $Z\left(f_{1}\right), \ldots, Z\left(f_{s}\right)$ are the irreducible components of codimension 1 of $\operatorname{rep}(Q, \mathbf{d}) \backslash \mathrm{Gl}(\mathbf{d}) \star T$, where $\mathrm{Gl}(\mathbf{d}) \star T$ is the open orbit. It is easy to see that

$$
g \cdot f_{i}=\chi_{i}(g) \cdot f_{i}
$$

for $g \in \mathrm{Gl}(\mathbf{d})$, where $\chi_{i}: \mathrm{Gl}(\mathbf{d}) \rightarrow k^{*}$ is a character. A regular function with this property is called a semi-invariant. By [11], any semi-invariant is a scalar multiple of a monomial in $f_{1}, \ldots, f_{s}$, and $f_{1}, \ldots, f_{s}$ are algebraically independent. We denote by

$$
\mathcal{Z}_{Q, \mathbf{d}}=\left\{X \in \operatorname{rep}(Q, \mathbf{d}) ; f_{i}(X)=0, i=1, \ldots, s\right\}
$$

the closed subscheme of $\operatorname{rep}(Q, \mathbf{d})$ of common zeros of all non-constant semiinvariants. Obviously we have $\operatorname{codim} \mathcal{Z}_{Q, \mathbf{d}} \leq s$, and equality means that $\mathcal{Z}_{Q, \mathbf{d}}$ is a complete intersection.

Let $T_{1}, \ldots, T_{r}$ be pairwise non-isomorphic indecomposable representations of $Q$ such that $\operatorname{Ext}_{Q}^{1}\left(T_{i}, T_{j}\right)=0$ for any $i, j \leq r$. In [8] we showed that there is a positive integer $N$ such that $\mathcal{Z}_{Q, \mathbf{d}}$ is a complete intersection and irreducible for any dimension vector $\mathbf{d}=\sum_{i=1}^{r} \lambda_{i} \operatorname{dim} T_{i}$ with $\lambda_{i} \geq N, i=1,2, \ldots, r$. Now our goal is to prove that $N$ is quite small in case $Q$ is tame; i.e., every connected component $\Delta$ of $Q$ is either a Dynkin quiver or an extended Dynkin quiver. Our methods are completely different.

Assume that $Q$ is tame, and set

$$
N(Q)=\max N(\Delta)
$$


where $\Delta$ ranges over the connected components of $Q$ and where

$$
N(\Delta)= \begin{cases}1 & \text { if }|\Delta|=\mathbb{A}_{m} \text { or } \widetilde{\mathbb{A}}_{m}, \\ 2 & \text { if }|\Delta|=\mathbb{D}_{m}, \mathbb{E}_{6}, \mathbb{E}_{7} \text { or } \mathbb{E}_{8}, \\ 3 & \text { if }|\Delta|=\widetilde{\mathbb{D}}_{m}, \widetilde{\mathbb{E}}_{6}, \widetilde{\mathbb{E}}_{7} \text { or } \widetilde{\mathbb{E}}_{8},\end{cases}
$$

and $|\Delta|$ denotes the underlying non-oriented graph of the quiver $\Delta$. Note that $N(K) \leq N(Q)$ for any subquiver $K$ of $Q$.

Theorem 1.1. Suppose $Q$ is tame. Let $T_{1}, \ldots, T_{r}$ be pairwise non-isomorphic indecomposable representations of $Q$ such that $\operatorname{Ext}_{Q}^{1}\left(T_{i}, T_{j}\right)=0$ for any $i, j \leq r$. Choose positive integers $\lambda_{1}, \ldots, \lambda_{r}$ and set $\lambda=\min \lambda_{i}, \mathbf{d}=\sum_{i=1}^{r} \lambda_{i} \operatorname{dim} T_{i}$. Then $\mathcal{Z}_{Q, \mathbf{d}}$ is

(i) a complete intersection provided $\lambda \geq N(Q)$,

(ii) irreducible provided $\lambda \geq N(Q)+1$.

Note that the case of a Dynkin quiver of type $\mathbb{A}_{n}$ has been treated by Chang and Weyman in [5].

In case $k$ is the field $\mathbb{C}$ of complex numbers, the fact that $\mathcal{Z}_{Q, \mathbf{d}}$ is a complete intersection implies that $\operatorname{rep}(Q, \mathbf{d})$ is cofree as a representation of the subgroup $\mathrm{Sl}(\mathbf{d})=\prod_{i=1}^{n} \mathrm{Sl}\left(d_{i}\right)$ of $\mathrm{Gl}(\mathbf{d})$; i.e., $\mathbb{C}[\operatorname{rep}(Q, \mathbf{d})]$ is a free module over the ring $\mathbb{C}[\operatorname{rep}(Q, \mathbf{d})]^{\overline{S l}(\mathbf{d})}$ of $\mathrm{Sl}(\mathbf{d})$-invariant polynomials $[13, \S 17],[8]$.

Example. Let us consider the quiver

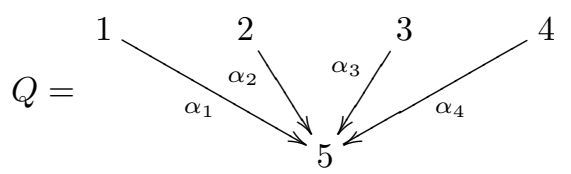

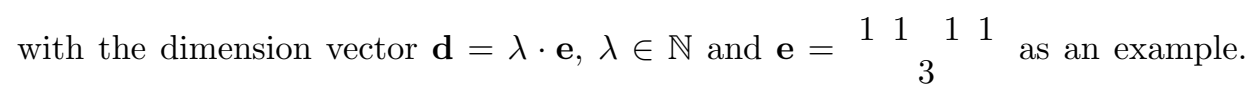

There is an indecomposable representation $T_{1}$ in $\operatorname{rep}(Q, \mathbf{e})$, whose orbit is open. The complement of the open orbit of $T=T_{1}^{\lambda}$ in $\operatorname{rep}(Q, \mathbf{d})$ has 4 components of codimension 1 , defined by

$$
\operatorname{det}\left(X\left(\alpha_{1}\right) \cdots \widehat{X\left(\alpha_{j}\right)} \cdots X\left(\alpha_{4}\right)\right)=0
$$

$j=1,2,3,4$, where the hat means "omit $X\left(\alpha_{j}\right)$ ". Using the results developed later, we know that $X$ belongs to $\mathcal{Z}_{Q, \mathbf{d}}$ if and only if $X$ either contains the simple projective $P_{5}$ or else the direct sum $\bigoplus_{j=1}^{4} P_{j}$ of the two-dimensional projectives associated to the vertices $1, \ldots, 4$ as a direct summand. It is easy to check that

- $\mathcal{Z}_{Q, \mathbf{e}}$ is irreducible of codimension 2 ,

- $\mathcal{Z}_{Q, 2 \mathbf{e}}$ has two irreducible components of codimension 3 and 4 , respectively,

- $\mathcal{Z}_{Q, 3 \mathbf{e}}$ has two irreducible components of codimension 4 ,

- $\mathcal{Z}_{Q, \lambda \cdot \mathbf{e}}$ is irreducible of codimension 4 for $\lambda \geq 4$. 


\section{Notations and preliminaries}

The varieties considered in this paper are locally closed subsets of a $k$-vector space. If $\mathcal{A} \subseteq \mathcal{B}$ are two such varieties and $\mathcal{B}$ is irreducible, we denote by $\operatorname{codim}_{\mathcal{B}} \mathcal{A}$ the codimension of $\mathcal{A}$ in $\mathcal{B}$. In case $\mathcal{B}=\operatorname{rep}(Q, \mathbf{d})$, we omit the subscript $\mathcal{B}$.

We will assume throughout that the representation $T=\bigoplus_{i=1}^{r} T_{i}^{\lambda_{i}}$ is sincere, i.e., $T(l) \neq 0$ for any $l \in Q_{0}$. As the full subquiver $K$ of $Q$ which supports $T$ is still tame with $N(K) \leq N(Q)$, this is no restriction. The assumption excludes oriented cycles as subquivers of $Q$. Indeed, a sincere representation of an oriented cycle cannot have an open orbit.

The Euler form of $Q$ is the $\mathbb{Z}$-bilinear form on $\mathbb{Z}^{Q_{0}}$ defined by

$$
\langle\mathbf{d}, \mathbf{e}\rangle=\sum_{i \in Q_{0}} d_{i} e_{i}-\sum_{\alpha \in Q_{1}} d_{t \alpha} e_{h \alpha} .
$$

For $X \in \operatorname{rep}(Q, \mathbf{d}), Y \in \operatorname{rep}(Q, \mathbf{e})$ it can be computed as

$$
\langle\mathbf{d}, \mathbf{e}\rangle=[X, Y]-{ }^{1}[X, Y],
$$

where

$$
[X, Y]=\operatorname{dim}_{k} \operatorname{Hom}_{Q}(X, Y) \quad \text { and } \quad{ }^{1}[X, Y]=\operatorname{dim}_{k} \operatorname{Ext}_{Q}^{1}(X, Y) .
$$

The quadratic form

$$
q(\mathbf{d})=\langle\mathbf{d}, \mathbf{d}\rangle
$$

associated with the Euler form is the Tits form of $Q$. It is positive semi-definite as $Q$ is tame and positive definite if $Q$ does not contain extended Dynkin diagrams.

We follow Schofield [12] in order to describe the semi-invariants of $\operatorname{rep}(Q, \mathbf{d})$ : For a representation $U$ of $Q$, the right perpendicular category $U^{\perp}$ is the full subcategory of $\operatorname{rep}(Q)$ whose objects are

$$
\left\{Y ;[U, Y]={ }^{1}[U, Y]=0\right\} .
$$

Dually, ${ }^{\perp} U$ has as objects

$$
\left\{Z ;[Z, U]={ }^{1}[Z, U]=0\right\} .
$$

Note that $U^{\perp}={ }^{\perp}(\tau U)$, where $\tau$ is the Auslander-Reiten translation for all nonprojective indecomposable direct summands of $U$ and $\tau\left(P_{l}\right)=I_{l}$, where $P_{l}$ and $I_{l}$ are the projective and injective indecomposable representations associated to the vertex $l \in Q_{0}$, respectively. If ${ }^{1}[U, U]=0$, the category $U^{\perp}$ is equivalent to the category of representations of a quiver with $n-\sigma(U)$ vertices.

Thus $T^{\perp}$ contains $n-r$ simple objects if $T=\bigoplus_{i=1}^{r} T_{i}^{\lambda_{i}}$ is a representation of $Q$ as in the statement of the theorem. If $S$ is one of them, the set

$$
\{X \in \operatorname{rep}(Q, \mathbf{d}) ;[X, S] \neq 0\}
$$

is a component of codimension 1 of the complement

$$
\operatorname{rep}(Q, \mathbf{d}) \backslash \mathrm{Gl}(\mathbf{d}) \star T .
$$


Non-isomorphic simple objects lead to distinct components, and all components of codimension 1 are obtained in this way. Thus $\mathcal{Z}_{Q, \mathbf{d}}$ is the zero set of $n-r$ (algebraically independent) polynomials. From now on, we will denote the underlying reduced variety of $\mathcal{Z}_{Q, \mathbf{d}}$ by the same symbol. This will cause no confusion since we are only interested in the irreducibility and the dimension of $\mathcal{Z}_{Q, \mathbf{d}}$. We have the following descriptions:

$$
\begin{aligned}
\mathcal{Z}_{Q, \mathbf{d}} & =\left\{X \in \operatorname{rep}(Q, \mathbf{d}) ;[X, S] \neq 0 \text { for all simple objects } S \in T^{\perp}\right\} \\
& =\left\{X \in \operatorname{rep}(Q, \mathbf{d}) ;\left[S^{\prime}, X\right] \neq 0 \text { for all simple objects } S^{\prime} \in{ }^{\perp} T\right\} .
\end{aligned}
$$

The material presented here can be found in [12]; compare also [8]. In order to obtain part (i) of our theorem it suffices to prove $\operatorname{codim} \mathcal{Z}_{Q, \mathbf{d}} \geq n-r$.

Fix a sink $z \in Q_{0}$; i.e., a vertex $z$ which is the head of some arrows $\alpha_{j}: y_{j} \rightarrow z$, $j=1, \ldots, s$, but the tail of none. The vertices $y_{1}, \ldots, y_{s}$ need not be distinct. Let $E$ be the simple projective supported at $z$. By $\bar{Q}$ we denote the full subquiver of $Q$ with $\bar{Q}_{0}=Q_{0} \backslash\{z\}$ and by $\overline{\mathbf{d}}$ the restriction of $\mathbf{d}$ to $\bar{Q}_{0}$. Note that the orbit of the restriction $\bar{T}=\bigoplus_{i=1}^{r} \bar{T}_{i}^{\lambda_{i}}$ to $\bar{Q}$ is open in $\operatorname{rep}(\bar{Q}, \overline{\mathbf{d}})$. As $E$ is the simple projective supported at $z$, we have

$$
E^{\perp}=\{X \in \operatorname{rep}(Q) ; X(z)=0\},
$$

which we identify with $\operatorname{rep}(\bar{Q})$. There is a short exact sequence

$$
0 \rightarrow E^{d_{z}} \rightarrow T \rightarrow \bar{T} \rightarrow 0
$$

Considering the long exact sequence of Hom's and Ext ${ }^{1}$ 's from it, we find that $E^{\perp} \cap T^{\perp}=E^{\perp} \cap \bar{T}^{\perp}=\bar{T}^{\perp \bar{Q}}$.

We decompose $\mathcal{Z}_{Q, \mathbf{d}}$ as a disjoint union

$$
\mathcal{Z}_{Q, \mathbf{d}}=\mathcal{Z}_{Q, \mathbf{d}}^{\prime} \cup \mathcal{Z}_{Q, \mathbf{d}}^{\prime \prime}
$$

where

$$
\mathcal{Z}_{Q, \mathbf{d}}^{\prime}=\left\{X \in \mathcal{Z}_{Q, \mathbf{d}} ;[X, E]=0\right\} \quad \text { and } \quad \mathcal{Z}_{Q, \mathbf{d}}^{\prime \prime}=\left\{X \in \mathcal{Z}_{Q, \mathbf{d}} ;[X, E] \neq 0\right\} .
$$

We will estimate the codimensions of $\mathcal{Z}_{Q, \mathbf{d}}^{\prime}$ and $\mathcal{Z}_{Q, \mathbf{d}}^{\prime \prime}$ in $\operatorname{rep}(Q, \mathbf{d})$ separately.

Throughout the article, $T=\bigoplus_{i=1}^{r} T_{i}^{\lambda_{i}}$ will denote a sincere representation of a tame quiver $Q$, and we set $\lambda=\min \lambda_{i} \geq 1$ and $\operatorname{dim} T=\mathbf{d}$.

\section{The variety $\mathcal{Z}_{Q, \mathrm{~d}}^{\prime \prime}$}

Proposition 3.1. A representation $X$ in $\mathcal{Z}_{Q, \mathbf{d}}$ belongs to $\mathcal{Z}_{Q, \mathbf{d}}^{\prime \prime}$ if and only if

(i) the restriction $\bar{X}$ to $\bar{Q}$ lies in $\mathcal{Z}_{\bar{Q}, \overline{\mathbf{d}}}$

and

(ii) $\operatorname{rank}\left(X\left(\alpha_{1}\right) \cdots X\left(\alpha_{s}\right)\right)<d_{z}$. 
In particular,

$$
\operatorname{codim} \mathcal{Z}_{Q, \mathbf{d}}^{\prime \prime}=\operatorname{codim}_{\operatorname{rep}(\bar{Q}, \overline{\mathbf{d}})} \mathcal{Z}_{\bar{Q}, \overline{\mathbf{d}}}+\max \left(0,\left(\sum_{j=1}^{s} d_{y_{j}}\right)-d_{z}+1\right)
$$

Proof. The second condition just says that $E$ is a direct summand of $X$, or equivalently that $[X, E] \neq 0$. A representation $X=X^{\prime} \oplus E$ belongs to $\mathcal{Z}_{Q, \mathbf{d}}$ if and only if

$$
[X, S]=\left[X^{\prime}, S\right]+[E, S]>0
$$

for any simple object $S \in T^{\perp}$. Equivalently,

$$
\left[X^{\prime}, S\right]>0
$$

holds for any simple representation $S \in T^{\perp}$ with $[E, S]=\operatorname{dim} S(z)=0$. These are precisely the simple objects of $\bar{T}^{\perp} \bar{Q}$, and moreover we have

$$
\left[X^{\prime}, S\right]=\left[\overline{X^{\prime}}, S\right]=[\bar{X}, S]>0
$$

since $S(z)=0$.

As for the statement about $\operatorname{codim} \mathcal{Z}_{Q, \mathbf{d}}^{\prime \prime}$, observe that, in case $d_{z}>\sum_{j=1}^{s} d_{y_{j}}$, any $d_{z} \times \sum_{j=1}^{s} d_{y_{j}}$-matrix has rank less than $d_{z}$, whereas for $d_{z} \leq \sum_{j=1}^{s} d_{y_{j}}$, the subvariety

$$
\mathcal{N}_{\mathbf{d}}=\left\{A \in \operatorname{Mat}\left(d_{z} \times \sum_{j=1}^{s} d_{y_{j}}\right) ; \operatorname{rank} A<d_{z}\right\}
$$

is of codimension $\left(\sum_{j=1}^{s} d_{y_{j}}\right)-d_{z}+1$.

Corollary 3.2. Suppose that $\lambda \geq N(Q)$ and that $E$ is not a direct summand of $T$.

(i) We have

$$
\operatorname{codim} \mathcal{Z}_{Q, \mathbf{d}}^{\prime \prime}-n+\sigma(T) \geq \operatorname{codim} \mathcal{Z}_{\bar{Q}, \overline{\mathbf{d}}}-(n-1)+\sigma(\bar{T}) .
$$

(ii) If moreover $d_{z}<\sum_{j=1}^{s} d_{y_{j}}$, we have

$$
\operatorname{codim} \mathcal{Z}_{Q, \mathbf{d}}^{\prime \prime}-n+\sigma(T) \geq \operatorname{codim} \mathcal{Z}_{\bar{Q}, \overline{\mathbf{d}}}-(n-1)+\sigma(\bar{T})+\lambda-N(Q) .
$$

In order to prove this result, we need some information about the number $\sigma(\bar{T})$ of pairwise non-isomorphic indecomposables occurring as direct summands of $\bar{T}$. We start by estimating $\sigma(\bar{U})$ for an indecomposable representation $U$ :

Lemma 3.3. For an indecomposable representation $U \neq E$ of $Q$, we have

$$
\sigma(\bar{U}) \leq 1+N(Q) \cdot\left(\left(\sum_{j=1}^{s} \operatorname{dim} U\left(y_{j}\right)\right)-\operatorname{dim} U(z)\right) .
$$


Proof. As $U$ is indecomposable, we may assume $Q$ to be connected. We use the following abbreviations:

$$
\operatorname{dim} U(z)=u, \quad \operatorname{dim} U\left(y_{j}\right)=u_{j}, j=1, \ldots, s, \quad u^{\prime}=\left(\sum_{j=1}^{s} u_{j}\right)-u .
$$

Note that $u^{\prime} \geq 0$ since $U$ is indecomposable and $U \neq E$. If $u=0, \bar{U}=U$ is indecomposable and $\sigma(\bar{U})=1$. In case $u^{\prime}=0$, the map

$$
\left[U\left(\alpha_{1}\right), \cdots, U\left(\alpha_{s}\right)\right]: \bigoplus_{j=1}^{s} U\left(y_{j}\right) \rightarrow U(z)
$$

is an isomorphism, and again $\bar{U}$ is indecomposable. Thus we may suppose $u>0$ and $u^{\prime}>0$.

Recall that the value of the Tits form $q(\operatorname{dim} U)$ equals 0 or 1 , as $Q$ is tame. We compute:

$$
\begin{aligned}
q(\operatorname{dim} U-\operatorname{dim} E) & =q(\operatorname{dim} U)+q(\operatorname{dim} E)-\langle\operatorname{dim} U, \operatorname{dim} E\rangle-\langle\operatorname{dim} E, \operatorname{dim} U\rangle \\
& =q(\operatorname{dim} U)+q(\operatorname{dim} E)+u^{\prime}-u \leq 2+u^{\prime}-u .
\end{aligned}
$$

As $q$ is positive definite or positive semi-definite in case $Q$ is a Dynkin quiver or an extended Dynkin quiver, respectively, we obtain:

$$
u \leq \begin{cases}u^{\prime}+2 \leq 2 u^{\prime}+1 & \text { if } Q \text { is an extended Dynkin quiver, } \\ u^{\prime}+1 & \text { if } Q \text { is a Dynkin quiver. }\end{cases}
$$

Now clearly $\bar{U}$ has at most $\sum_{j=1}^{s} u_{j}$ indecomposable direct summands, and thus

$$
\sigma(\bar{U}) \leq \sum_{j=1}^{s} u_{j}=u+u^{\prime} \leq \begin{cases}1+3 u^{\prime} & \text { if } Q \text { is an extended Dynkin quiver, } \\ 1+2 u^{\prime} & \text { if } Q \text { is a Dynkin quiver, }\end{cases}
$$

which proves the lemma except in case $|Q|=\mathbb{A}_{n}$ or $|Q|=\widetilde{\mathbb{A}}_{n-1}$.

If $|Q|=\mathbb{A}_{n}$, we have $u \leq 1$ and hence $\sigma(\bar{U}) \leq 1+u^{\prime}$. In case $|Q|=\widetilde{\mathbb{A}}_{n-1}$, the number of indecomposable (possible isomorphic) direct summands in a decomposition of $\bar{U}$ is at most $1+u^{\prime}$. This can be seen by inspecting the list of indecomposable representations of $Q$. Such representations are string or band representations, and they are described by words (non-oriented paths) in $Q$ (see [4] for details).

Proof of Corollary 3.2. We set

$$
t_{i}^{\prime}=\left(\sum_{j=1}^{s} \operatorname{dim} T_{i}\left(y_{j}\right)\right)-\operatorname{dim} T_{i}(z), i=1, \ldots, r
$$

and

$$
t^{\prime}=\sum_{i=1}^{r} t_{i}^{\prime}
$$


Note that, by definition,

$$
\sum_{i=1}^{r} \lambda_{i} t_{i}^{\prime}=\left(\sum_{j=1}^{s} d_{y_{j}}\right)-d_{z}
$$

Our lemma implies:

$$
\begin{aligned}
\sigma(\bar{T}) & \leq \sum_{i=1}^{r} \sigma\left(\overline{T_{i}}\right) \leq r+N(Q) \cdot t^{\prime} \leq r+\left(\sum_{i=1}^{r} \lambda_{i} t_{i}^{\prime}\right)-(\lambda-N(Q)) \cdot t^{\prime} \\
& =\sigma(T)+\left(\sum_{j=1}^{s} d_{y_{j}}\right)-d_{z}-(\lambda-N(Q)) \cdot t^{\prime}
\end{aligned}
$$

Combining this with Proposition 3.1 we find that

$$
\begin{aligned}
\operatorname{codim} \mathcal{Z}_{Q, \mathbf{d}}^{\prime \prime}-n+\sigma(T) & =\operatorname{codim}_{\operatorname{rep}(\bar{Q}, \overline{\mathbf{d}})} \mathcal{Z}_{\bar{Q}, \overline{\mathbf{d}}}+\left(\sum_{j=1}^{s} d_{y_{j}}\right)-d_{z}+1-n+\sigma(T) \\
& \geq \operatorname{codim}_{\mathrm{rep}(\bar{Q}, \overline{\mathbf{d}})} \mathcal{Z}_{\bar{Q}, \overline{\mathbf{d}}}-(n-1)+\sigma(\bar{T})+(\lambda-N(Q)) \cdot t^{\prime} .
\end{aligned}
$$

As $t_{i}^{\prime} \geq 0$ for all $i$, this yields part (i) of Corollary 3.2. Part (ii) follows from the fact that $\sum_{i=1}^{r} \lambda_{i} t_{i}^{\prime}=\left(\sum_{j=1}^{s} d_{y_{j}}\right)-d_{z}>0$ implies $t_{i}^{\prime}>0$ for some $i$ and hence $t^{\prime}>0$.

\section{Reflection functors}

We define two new quivers $\widetilde{Q}$ and $Q^{\prime}: \widetilde{Q}$ is obtained from $Q$ by adding a vertex $z^{\prime}$ and arrows $\beta_{j}: z^{\prime} \rightarrow y_{j}, j=1, \ldots, s$. Deleting $z$ and $\alpha_{1}, \ldots, \alpha_{s}$ in $\widetilde{Q}$ yields $Q^{\prime}$. Note that $Q^{\prime}$ is tame as well. We denote by $E^{\prime}$ the simple injective representation of $Q^{\prime}$ supported at $z^{\prime}$.

We consider the reflection functor

$$
\mathcal{F}: \operatorname{rep}(Q) \rightarrow \operatorname{rep}\left(Q^{\prime}\right)
$$

associated with $z$. Recall that

$$
(\mathcal{F} X)(i)= \begin{cases}X(i) & i \neq z^{\prime} \\ \operatorname{ker}\left(\bigoplus X\left(y_{j}\right) \stackrel{\left[X\left(\alpha_{1}\right), \ldots, X\left(\alpha_{s}\right)\right]}{\longrightarrow} X(z)\right) & i=z^{\prime}\end{cases}
$$

and that

$$
(\mathcal{F} X)\left(\beta_{l}\right):(\mathcal{F} X)\left(z^{\prime}\right) \rightarrow(\mathcal{F} X)\left(y_{l}\right)=X\left(y_{l}\right)
$$

is the inclusion of $(\mathcal{F} X)\left(z^{\prime}\right)$ into $\bigoplus_{j=1}^{s} X\left(y_{j}\right)$ followed by the projection to $X\left(y_{l}\right)$ (see [1], [6]). The functor $\mathcal{F}$ restricts to an equivalence

$$
\mathcal{F}:(\operatorname{rep}(Q))^{\prime} \rightarrow\left(\operatorname{rep}\left(Q^{\prime}\right)\right)^{\prime}
$$


from the full subcategory $(\operatorname{rep}(Q))^{\prime}$ of $\operatorname{rep}(Q)$ whose objects do not contain $E$ as a direct summand, or equivalently have no non-trivial morphisms to $E$, to the full subcategory $\left(\operatorname{rep}\left(Q^{\prime}\right)\right)^{\prime}$ of $\operatorname{rep}\left(Q^{\prime}\right)$ whose objects do not contain $E^{\prime}$ as a direct summand.

Suppose that $E$ is neither a direct summand of $T$ nor an element of $T^{\perp}$. This implies that $[T, E]=0$ and ${ }^{1}[T, E]>0$ and thus the vector $\mathbf{d}^{\prime} \in \mathbb{Z}^{Q_{0}^{\prime}}$, where $Q_{0}^{\prime}$ denotes the set of vertices of $Q^{\prime}$, defined by

$$
d_{x}^{\prime}= \begin{cases}d_{x}, & x \neq z^{\prime} \\ \left(\sum_{j=1}^{s} d_{y_{j}}\right)-d_{z}, & x=z^{\prime}\end{cases}
$$

has positive entries. Indeed, we have

$$
d_{z^{\prime}}^{\prime}=\left(\sum_{j=1}^{s} d_{y_{j}}\right)-d_{z}=-\langle\mathbf{d}, \operatorname{dim} E\rangle=-[T, E]+{ }^{1}[T, E]>0 .
$$

Note that in fact we have $d_{z^{\prime}}^{\prime} \geq \lambda$ as ${ }^{1}\left[T_{i}, E\right]>0$ for some $i$ implies ${ }^{1}[T, E] \geq \lambda_{i} \geq$ $\lambda$. We let $\widetilde{\mathbf{d}}$ be the dimension vector for $\widetilde{Q}$ which coincides with $\mathbf{d}$ on $Q_{0}$ and with $\mathbf{d}^{\prime}$ on $Q_{0}^{\prime}$.

As $E$ is not a direct summand of $T$, the latter belongs to $(\operatorname{rep} Q)^{\prime}$. Therefore $\mathcal{F} T$ lies in $\left(\operatorname{rep} Q^{\prime}\right)^{\prime}$, and we have $\operatorname{dim} \mathcal{F} T=\mathbf{d}^{\prime},{ }^{1}[\mathcal{F} T, \mathcal{F} T]={ }^{1}[T, T]=0$, and thus $\mathbf{d}^{\prime}$ is prehomogeneous. Choose $T^{\prime}$ in the open orbit of $\operatorname{rep}\left(Q^{\prime}, \mathbf{d}^{\prime}\right)$. As $T^{\prime}$ is isomorphic to $\mathcal{F} T$, we have $T^{\prime}=\bigoplus_{i=1}^{r}\left(T_{i}^{\prime}\right)^{\lambda_{i}}$ with $T_{i}^{\prime}$ indecomposable, pairwise non-isomorphic and ${ }^{1}\left[T_{i}^{\prime}, T_{j}^{\prime}\right]=0$ for all $i, j$. Moreover, we know $T^{\perp} \subseteq(\operatorname{rep} Q)^{\prime}$, as $E$ does not belong to $T^{\perp}$, and $\left(T^{\prime}\right)^{\perp} \subseteq\left(\operatorname{rep} Q^{\prime}\right)^{\prime}$, as $d_{z^{\prime}}^{\prime}=\left[T^{\prime}, E^{\prime}\right]>0$. We conclude that $\left(T^{\prime}\right)^{\perp}$ is equivalent to $\mathcal{F}\left(T^{\perp}\right)$, the category of representations of a quiver with $n-r$ vertices. Hence $\mathcal{Z}_{Q^{\prime}, \mathbf{d}^{\prime}}$ is given by $n-r$ equations as well. We decompose $\mathcal{Z}_{Q^{\prime}, \mathbf{d}^{\prime}}$ as a disjoint union $\mathcal{Z}_{Q^{\prime}, \mathbf{d}^{\prime}}=\mathcal{W}_{Q^{\prime}, \mathbf{d}^{\prime}}^{\prime} \cup \mathcal{W}_{Q^{\prime}, \mathbf{d}^{\prime}}^{\prime \prime}$, where

$$
\mathcal{W}_{Q^{\prime}, \mathbf{d}^{\prime}}^{\prime}=\left\{X^{\prime} \in \mathcal{Z}_{Q^{\prime}, \mathbf{d}^{\prime}} ;\left[E^{\prime}, X^{\prime}\right]=0\right\}
$$

and

$$
\mathcal{W}_{Q^{\prime}, \mathbf{d}^{\prime}}^{\prime \prime}=\left\{X^{\prime} \in \mathcal{Z}_{Q^{\prime}, \mathbf{d}^{\prime}} ;\left[E^{\prime}, X^{\prime}\right] \neq 0\right\}
$$

Proposition 4.1. Suppose $E$ is neither a direct summand of $T$ nor an element of $T^{\perp}$. Then we have and

(i) $\operatorname{codim} \mathcal{Z}_{Q, \mathbf{d}}^{\prime}=\operatorname{codim}_{\mathrm{rep}\left(Q^{\prime}, \mathbf{d}^{\prime}\right)} \mathcal{W}_{Q^{\prime}, \mathbf{d}^{\prime}}^{\prime}$

(ii) $\mathcal{Z}_{Q, \mathbf{d}}^{\prime}$ is irreducible if $\mathcal{W}_{Q^{\prime}, \mathbf{d}^{\prime}}^{\prime}$ has this property.

Proof. By construction, $X$ belongs to $\mathcal{Z}_{Q, \mathbf{d}}^{\prime}$ if and only if $\mathcal{F} X$ is isomorphic to some $X^{\prime} \in \mathcal{W}_{Q^{\prime}, \mathbf{d}^{\prime}}^{\prime}$, but unfortunately the functor $\mathcal{F}$ cannot be made into a regular map from

$$
\operatorname{rep}(Q, \mathbf{d})^{\prime}=\{X \in \operatorname{rep}(Q, \mathbf{d}) ;[X, E]=0\}
$$


to

$$
\operatorname{rep}\left(Q^{\prime}, \mathbf{d}^{\prime}\right)^{\prime}=\left\{X^{\prime} \in \operatorname{rep}\left(Q^{\prime}, \mathbf{d}^{\prime}\right) ;\left[E^{\prime}, X^{\prime}\right]=0\right\} .
$$

We use the following détour (compare [7] and Section 4.2 in [3]): The set

$$
\begin{array}{r}
\left\{X \in \operatorname{rep}(\widetilde{Q}, \widetilde{\mathbf{d}}) ; \sum_{j=1}^{s} X\left(\alpha_{j}\right) X\left(\beta_{j}\right)=0,\left[X\left(\beta_{1}\right), \ldots, X\left(\beta_{s}\right)\right]^{t}\right. \text { injective, } \\
\left.\left[X\left(\alpha_{1}\right), \ldots, X\left(\alpha_{s}\right)\right] \text { surjective }\right\}
\end{array}
$$

is a principal $\mathrm{Gl}\left(d_{z^{\prime}}^{\prime}\right)$-bundle over $\operatorname{rep}(Q, \mathbf{d})^{\prime}$ and a principal $\mathrm{Gl}\left(d_{z}\right)$-bundle over $\operatorname{rep}\left(Q^{\prime}, \mathbf{d}^{\prime}\right)^{\prime}$ via the projections $\pi$ and $\pi^{\prime}$ deleting $z^{\prime}$ and $z$, respectively. Hence the claim follows from $\pi^{-1}\left(\mathcal{Z}_{Q, \mathbf{d}}^{\prime}\right)=\left(\pi^{\prime}\right)^{-1}\left(\mathcal{W}_{Q^{\prime}, \mathbf{d}^{\prime}}^{\prime}\right)$.

\section{Proof of Theorem 1.1}

We proceed by induction on the number $n$ of vertices of $Q$. We may assume the theorem to be true for $\mathcal{Z}_{\bar{Q}}, \overline{\mathbf{d}}$. First we treat the cases that

(i) $E$ is a direct summand of $T$ and

(ii) $E$ belongs to $T^{\perp}$.

In both cases, we have that $E$ is a direct summand of $X$ for all $X \in \mathcal{Z}_{Q \text {,d }}$; i.e., $\mathcal{Z}_{Q, \mathbf{d}}^{\prime \prime}=\mathcal{Z}_{Q, \mathbf{d}}$. Indeed, in case (i) this follows from the fact that $\operatorname{Hom}_{Q}(E, T) \neq 0$, which is a closed condition. In case (ii), $E$ is a simple object in $T^{\perp}$.

As any direct summand $T_{i} \nsucceq E$ of $T$ belongs to ${ }^{\perp} E$, we have

$$
\operatorname{dim} T_{i}(z)-\sum_{j=1}^{s} \operatorname{dim} T_{i}\left(y_{j}\right)=\left\langle\operatorname{dim} T_{i}, \operatorname{dim} E\right\rangle=\left[T_{i}, E\right]-{ }^{1}\left[T_{i}, E\right]=0
$$

By Lemma 3.3, $\overline{T_{i}}$ is indecomposable, and therefore

$$
\sigma(\bar{T})= \begin{cases}r-1 & \text { in case (i) } \\ r & \text { in case (ii) }\end{cases}
$$

The induction hypothesis together with Corollary 3.2 implies the first part of our theorem. We conclude from Proposition 3.1 that $\mathcal{Z}_{Q, \mathbf{d}} \simeq \mathcal{Z}_{\bar{Q}, \overline{\mathbf{d}}} \times \mathcal{N}_{\mathbf{d}}$, where

$$
\mathcal{N}_{\mathbf{d}}=\left\{A \in \operatorname{Mat}\left(d_{z} \times \sum_{j=1}^{s} d_{y_{j}}\right) ; \operatorname{rank} A<d_{z}\right\} .
$$

The second part follows from the fact that the set $\mathcal{N}_{\mathbf{d}}$ is irreducible in case $d_{z} \geq$ $\sum_{j=1}^{s} d_{y_{j}}$.

(iii) Finally, suppose that $E$ is neither a direct summand of $T$ nor does it belong to $T^{\perp}$, or equivalently that $d_{z}<\sum_{j=1}^{s} d_{y_{j}}$. Using Corollary 3.2 and its dual, Proposition 4.1 and remembering that the codimension of any irreducible 
component of $\mathcal{Z}_{Q, \mathbf{d}}$ is at most $n-r$, we see that the theorem is true for $\mathcal{Z}_{Q, \mathbf{d}}$ if and only if it holds for $\mathcal{Z}_{Q^{\prime}, \mathbf{d}^{\prime}}$.

In case either $T$ contains a preprojective direct summand or $T^{\perp}$ a preprojective representation, we may apply a series of reflection functors until we reach the situation that a simple projective either is a direct summand of $T$ or else belongs to $T^{\perp}$, and we can reduce by (i) or (ii). This finishes the proof in case $Q$ is of finite representation type as any indecomposable representation is preprojective.

If $Q$ is not representation finite, we are left with the situation that no preprojective representation is a direct summand of $T$ nor an element of $T^{\perp}$. Dually, we may assume $T$ does not contain a preinjective direct summand either. Indeed, suppose a simple injective representation $E^{\prime}$ is a direct summand of $T$ or belongs to ${ }^{\perp} T$, a situation we will reach after a series of (inverse) reflection functors. Then apply the dual of the first or the second reduction step above; recall that $\mathcal{Z}_{Q, \mathbf{d}}$ has a dual description as

$$
\mathcal{Z}_{Q, \mathbf{d}}=\left\{X \in \operatorname{rep}(Q, \mathbf{d}) ;\left[S^{\prime}, X\right] \neq 0 \text { for all simple objects } S^{\prime} \in{ }^{\perp} T\right\} .
$$

The following lemma finishes the proof of Theorem 1.1.

Lemma 5.1. Let $Q$ be an extended Dynkin quiver. Suppose $T$ is a regular representation with an open orbit. Then $T^{\perp}$ contains a non-zero preprojective representation.

Proof. Consider a Bongartz completion $\widetilde{T}$ for $T$ [2]; i.e., an exact sequence

$$
0 \rightarrow k Q \rightarrow \widetilde{T} \rightarrow \bigoplus_{i=1}^{r} T_{i}^{\nu_{i}} \rightarrow 0
$$

for which the induced map

$$
\operatorname{Hom}_{Q}\left(T_{l}, \bigoplus_{i=1}^{r} T_{i}^{\nu_{i}}\right) \rightarrow \operatorname{Ext}_{Q}^{1}\left(T_{l}, k Q\right)
$$

is surjective for $l=1, \ldots, r$. There is a $\mathbb{Z}$-linear map $\partial: \mathbb{Z}^{Q_{0}} \rightarrow \mathbb{Z}$, called defect, such that any indecomposable representation $Y$ of $Q$ is preprojective, regular and preinjective if and only if $\partial(\operatorname{dim} Y)$ is negative, zero and positive, respectively (see for instance [10]). As $T$ is regular, $\partial \widetilde{T}=\partial k Q<0$ and therefore $\widetilde{T}$ contains an indecomposable preprojective direct summand $Y$, and $Y \in T^{\perp}$. Indeed, we have ${ }^{1}[T, Y]=0$ for all direct summands of $\widetilde{T}$ and $[T, Y]=0$ since $Y$ is preprojective and $T$ is regular [10, Theorem 3.6.5].

Example. Working out the following example, one can see that if $Q$ is not tame, it may happen that both $T$ and $T^{\perp}$ belong to the set of regular representations:

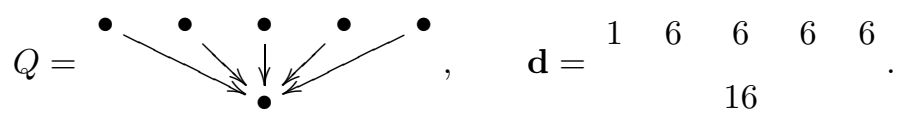


As $q(\mathbf{d})=1$, there exists an irreducible $T \in \operatorname{rep}(Q, \mathbf{d})$ having an open orbit. The simple objects in $T^{\perp}$ have dimension vectors

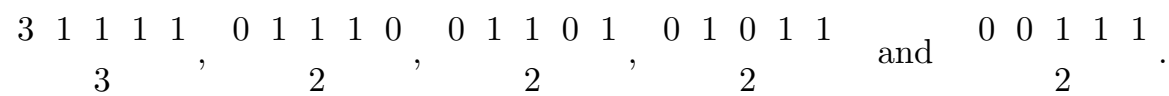

It is easy to check that these simple objects are regular representations of $Q$.

Acknowledgments. The second author gratefully acknowledges support from the Polish Scientific Grant KBN No. 5 PO3A 00821 and Foundation for Polish Science. He also thanks the Swiss Science Foundation, which gave him the opportunity to spend a year at the University of Berne.

\section{References}

[1] I. M. Bernstein, I. N. Gelfand and V. A. Ponomarev, Coxeter functors and Gabriel theorem, Russ. Math. Surveys 28 (1973), 17-32.

[2] K. Bongartz, Tilted algebras, in: Representations of Algebras, Lecture Notes in Math. 903 (1981), 26-38.

[3] K. Bongartz, Minimal singularities for representations of Dynkin quivers, Comment. Math. Helv. 63 (1994), 575-611.

[4] M. Butler and C. M. Ringel, Auslander-Reiten sequences with few middle terms and applications to string algebras, Comm. Algebra 15 (1987), 145-179.

[5] C. Chang and J. Weyman, Representations of quivers with free module of covariants, Preprint.

[6] P. Gabriel, Représentations indécomposables, Séminaire Bourbaki 1973/74, Lecture Notes in Math. 431 (1975), 143-169.

[7] H. Kraft and Ch. Riedtmann, Geometry of representations of quivers, LMS lecture notes 116 (1985), 109-147.

[8] Ch. Riedtmann and G. Zwara, On the zero set of semi-invariants for quivers, Preprint 2002, http://www.mat.uni.torun.pl/ gzwara/semiil.ps.

[9] C. M. Ringel, The rational invariants of tame quivers, Inv. Math. 58 (1980), 217-239.

[10] C. M. Ringel, Tame algebras and integral quadratic forms, Lecture Notes in Math. 1099, Springer Verlag, 1984.

[11] M. Sato and T. Kimura, A classification of irreducible prehomogeneous vector spaces and their relative invariants, Nagoya J. Math. 65 (1977), 1-155.

[12] A. Schofield, Semi-invariants of quivers, J. London Math. Soc. 43 (1991), 385-395.

[13] G. W. Schwarz, Lifting smooth homotopies of orbit spaces, Inst. Hautes Études Sci. Publ. Math. 51 (1980), 37-135.

Ch. Riedtmann

Mathematisches Institut

Universität Bern

Sidlerstr. 5

CH 3012 Bern

Switzerland

e-mail: christine.riedtmann@math-stat.unibe.ch

\section{G. Zwara}

Faculty of Mathematics and Computer Science Nicholas Copernicus University

Chopina $12 / 18$

PL-87-100 Toruń

Poland

(Received: October 23, 2002) 